\title{
Ionic Liquids as a Convenient New Medium for the Catalytic Asymmetric Dihydroxylation of Olefins Using a Recoverable and Reusable Osmium/Ligand
}

\author{
Luís C. Branco ${ }^{\mathrm{a}}$ and Carlos A. M. Afonso*b \\ ${ }^{a}$ REQUIMTE/CQFB, Departamento de Química, Faculdade de Ciências e Tecnologia, Universidade Nova de Lisboa, 2829-516 \\ Caparica, Portugal.; ${ }^{b}$ CQFM, Department of Chemical Engineering, Instituto Superior Técnico, 1049-001 Lisboa, Portugal E- \\ mail: carlosafonso@ist.utl.pt
}

\section{Content}

Figure 1. ${ }^{1} \mathrm{H}$ NMR spectra (in $d_{6}$-DMSO) of initial $\left[\mathrm{C}_{4}{ }^{\mathrm{mim}}\right]\left[\mathrm{PF}_{6}\right]$

Figure $2 .{ }^{13} \mathrm{C}$ NMR spectra (in $d_{6}$-DMSO) of initial $\left[\mathrm{C}_{4}{ }^{\mathrm{mim}}\right]\left[\mathrm{PF}_{6}\right]$

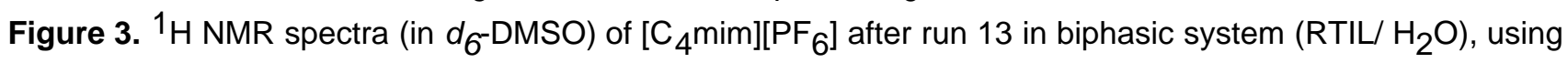
$\mathrm{K}_{3} \mathrm{Fe}(\mathrm{CN})_{6}$ as co-oxidan

Figure 4. ${ }^{13} \mathrm{C}$ NMR spectra (in $d_{6}$-DMSO) of [ $\mathrm{C}_{4}$ mim] $\left[\mathrm{PF}_{6}\right]$ after run 13 in biphasic system (RTIL/ $\mathrm{H}_{2} \mathrm{O}$ ), using $\mathrm{K}_{3} \mathrm{Fe}(\mathrm{CN})_{6}$ as co-oxidant

Figure 5. ${ }^{1} \mathrm{H}$ NMR spectra (in $d_{6}$-DMSO) of $\left[\mathrm{C}_{4}\right.$ mim] $\left[\mathrm{PF}_{6}\right]$ after run 13 in monophasic system $\left(\mathrm{RTIL} / \mathrm{H}_{2} \mathrm{O} / \mathrm{t}-\mathrm{BuOH}\right)$, using $\mathrm{K}_{3} \mathrm{Fe}(\mathrm{CN})_{6}$ as co-oxidant

Figure $6 .{ }^{13} \mathrm{C}$ NMR spectra (in $d_{6}$-DMSO) of $\left[\mathrm{C}_{4}\right.$ mim] $\left[\mathrm{PF}_{6}\right]$ after run 13 in monophasic system $\left(\mathrm{RTIL} / \mathrm{H}_{2} \mathrm{O} / \mathrm{t}-\mathrm{BuOH}\right)$, using $\mathrm{K}_{3} \mathrm{Fe}(\mathrm{CN})_{6}$ as co-oxidant

Figure 7. ${ }^{1} \mathrm{H}$ NMR spectra (in $d_{6}$-DMSO) of initial $\left[\mathrm{C}_{8} \mathrm{mim}\right]\left[\mathrm{PF}_{6}\right]$

Figure 8. ${ }^{13} \mathrm{C}$ NMR spectra (in $d_{6}$-DMSO) of initial $\left[\mathrm{C}_{8}\right.$ mim] $\left[\mathrm{PF}_{6}\right]$

Figure 9. ${ }^{1} \mathrm{H}$ NMR spectra (in $d_{6}$ DMSO) of $\left[\mathrm{C}_{8}\right.$ mim] $\left[\mathrm{PF}_{6}\right]$ after run 13 in biphasic system $\left(\mathrm{RTIL} / \mathrm{H}_{2} \mathrm{O}\right)$, using $\mathrm{K}_{3} \mathrm{Fe}(\mathrm{CN})_{6}$ as co-oxidant

Figure 10. ${ }^{13} \mathrm{C}$ NMR spectra (in $d_{6}$-DMSO) of $\left[\mathrm{C}_{8}\right.$ mim] $\left[\mathrm{PF}_{6}\right]$ after run 13 in biphasic system $\left(\mathrm{RTIL} / \mathrm{H}_{2} \mathrm{O}\right)$, using $\mathrm{K}_{3} \mathrm{Fe}(\mathrm{CN})_{6}$ as co-oxidant

Figure 11. ${ }^{1} \mathrm{H}$ NMR spectra (in $d_{6}$-DMSO) of $\left[\mathrm{C}_{8}\right.$ mim] $\left[\mathrm{PF}_{6}\right]$ after run 13 in monophasic system $\left(\mathrm{RTIL} / \mathrm{H}_{2} \mathrm{O} / \mathrm{t}-\mathrm{BuOH}\right)$, using $\mathrm{K}_{3} \mathrm{Fe}(\mathrm{CN})_{6}$ as co-oxidant

Figure 12. ${ }^{13} \mathrm{C}$ NMR spectra (in $d_{6}$-DMSO) of $\left[\mathrm{C}_{8}\right.$ mim] $\left[\mathrm{PF}_{6}\right]$ after run 13 in monophasic system $\left(\mathrm{RTIL} / \mathrm{H}_{2} \mathrm{O} / \mathrm{t}\right.$ $\mathrm{BuOH})$, using $\mathrm{K}_{3} \mathrm{Fe}(\mathrm{CN})_{6}$ as co-oxidant

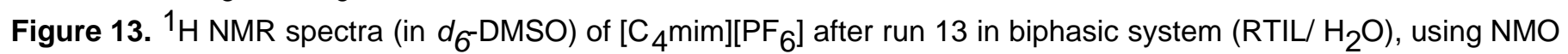
as co-oxidant

Figure 14. ${ }^{13} \mathrm{C}$ NMR spectra (in $d_{6}$-DMSO) of $\left[\mathrm{C}_{4}\right.$ mim] $\left[\mathrm{PF}_{6}\right]$ after run 13 in biphasic system $\left(\mathrm{RTIL} / \mathrm{H}_{2} \mathrm{O}\right)$, NMO as co-oxidant

Figure 15. ${ }^{1} \mathrm{H}$ NMR spectra (in $d_{6}$ DMSO) of $\left[\mathrm{C}_{4}\right.$ mim] $\left[\mathrm{PF}_{6}\right]$ after run 13 in monophasic system $\left(\mathrm{RTIL} / \mathrm{H}_{2} \mathrm{O} / \mathrm{t}-\mathrm{BuOH}\right)$, using NMO as co-oxidant

Figure 16. ${ }^{13} \mathrm{C}$ NMR spectra (in $d_{6}$-DMSO) of $\left[\mathrm{C}_{4}\right.$ mim] $\left[\mathrm{PF}_{6}\right]$ after run 13 in monophasic system $\left(\mathrm{RTIL} / \mathrm{H}_{2} \mathrm{O} / \mathrm{t}-\right.$ $\mathrm{BuOH}$ ), using $\mathrm{NMO}$ as co-oxidant

General: The following chemicals were purchased from commercial sources and used as supplied: styrene, $\alpha$ methylstyrene, 1-hexene, 1-methylcyclohexene, trans-stilbene, trans-5-decene, $\mathrm{K}_{2} \mathrm{CO}_{3}, \mathrm{~K}_{2} \mathrm{OsO}_{2}(\mathrm{OH})_{4}, \mathrm{~K}_{3}\left[\mathrm{Fe}(\mathrm{CN})_{6}\right]$, $\mathrm{N}$-methyl-morpholine oxide (NMO), (DHQD) ${ }_{2} \mathrm{PHAL},(\mathrm{DHQD})_{2} \mathrm{PYR}$ and $t$-butanol. All aqueous solutions were prepared using distilled water. The room temperature ionic liquids (RTIL) based on the methylimidazolium cation [mim] ${ }^{1}$, and on the tetra-alkyl-dimethylguanidinium cation $\left[(\mathrm{be})_{2} \mathrm{dmg}\right]^{2}$ were prepared following reported procedures. ${ }^{1} \mathrm{H}$, and ${ }^{13} \mathrm{C}$ NMR spectra were recorded on a Bruker AMX 400 spectrometer. Chemical shifts are reported downfield in parts per million (ppm) from a tetramethylsilane reference for ${ }^{1} \mathrm{H}$ and ${ }^{13} \mathrm{C}$ NMR. IR spectra were recorded on a FTIR instrument as thinlv disnersed films. 
Gas liquid chromatography (GLC) was carried out using He as carrier gas and chiral capillary column Supelco $\beta$-dex $120(30 \mathrm{~m} \times 0.25 \mathrm{~mm})$. HPLC analyses were performed using Chiralpak AD columm at $25{ }^{\circ} \mathrm{C}$. Flash chromatography was carried out using an MN-Kiesel-gel 60M gel (230-400 mesh ASTM, Art. 815381). All eluents were distilled prior to use. Preparative and analytical thin layer chromatography (TLC) was carried out using, respectively, MN Kieselgel G/UV254 (Art. 816320) glass-backed plates and MN Alugram, SIL G/UV 254 (Art. 818133). The plates were visualised using ultraviolet light $(254 \mathrm{~nm}$ ) or using phosphomolybdic acid.

\section{References}

[1] a) T. Kitazume, F. Zulfiqar, G. Tanaka, Green Chemistry, 2000, 2, 133; b) A. E Visser,. R. P. Swatloski, R. D. Rogers, Green Chemistry, 2000, 2, 1. c) L. C. Branco, J. N. Rosa, J. J. M. Ramos, C. A. M. Afonso, Chem. Eur. J. 2002, 8, 3671.

[2] N. M. M. Mateus, L. C. Branco, N. M. T. Lourenço, C. A. M. Afonso, Green Chem. 2003, 5 , 347.

\section{Comparison of Spectral Data $\left({ }^{1} \mathrm{H}\right.$ and ${ }^{13} \mathrm{C}$ NMR $)$ of the Ionic Liquid Between the Initial and Final Recycled Samples}

Figure 1. ${ }^{1} \mathrm{H}$ NMR spectra (in $d_{6}$-DMSO) of initial $\left[\mathrm{C}_{4}\right.$ mim] $\left[\mathrm{PF}_{6}\right]$

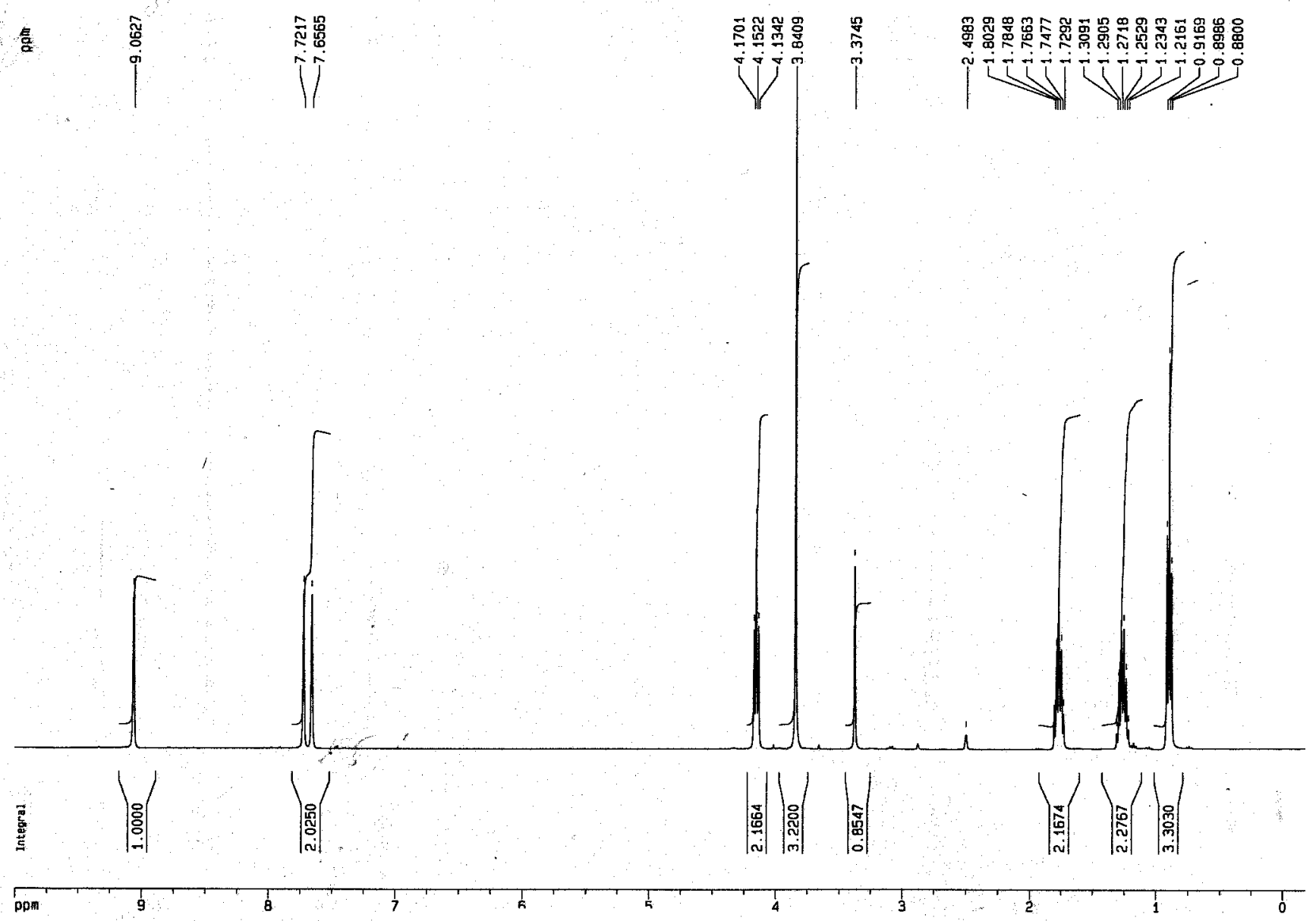


Figure $2 .{ }^{13} \mathrm{C}$ NMR spectra (in $d_{6}$-DMSO) of initial $\left[\mathrm{C}_{4} \mathrm{mim}\right]\left[\mathrm{PF}_{6}\right]$

镸

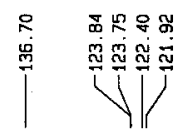

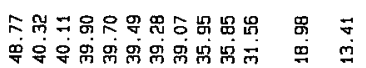

以s

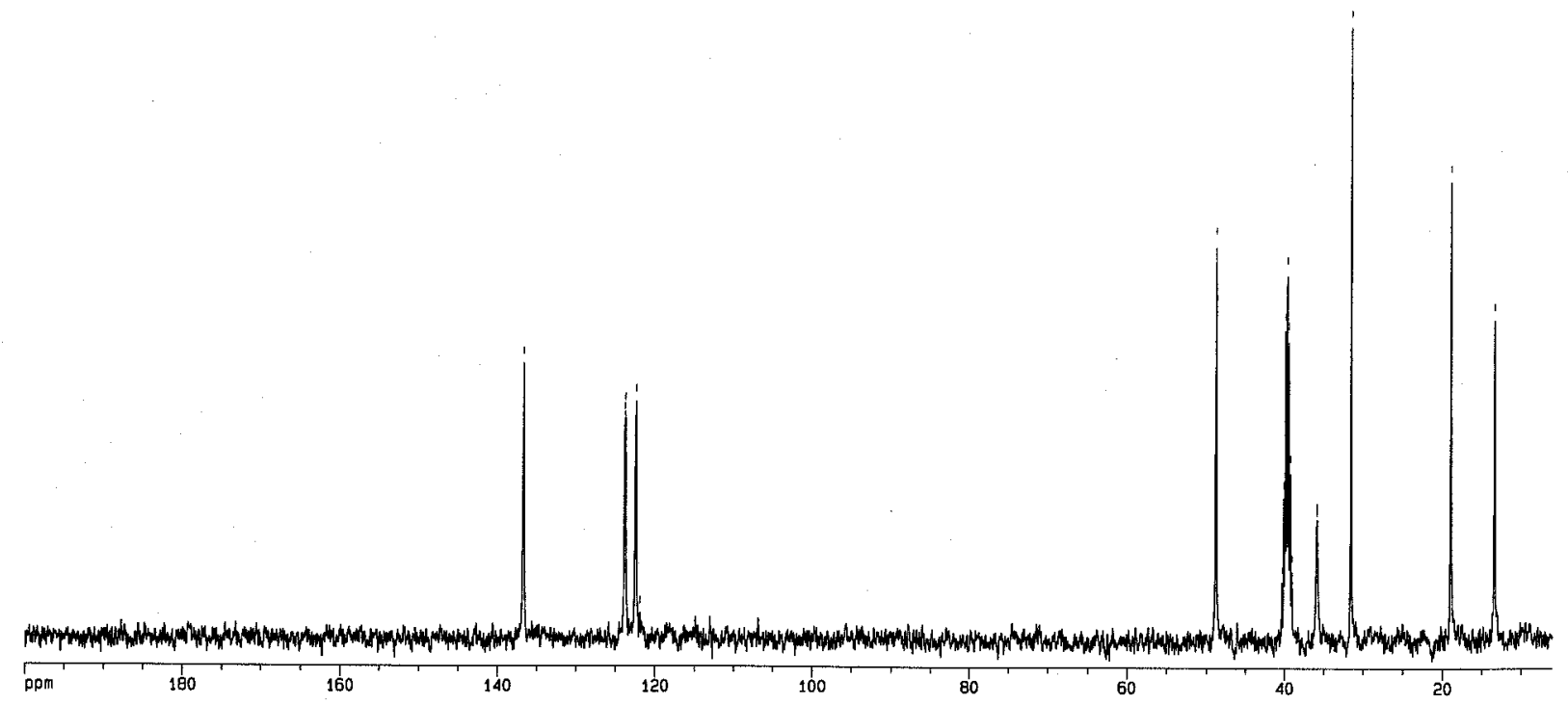

Figure 3. ${ }^{1} \mathrm{H}$ NMR spectra (in $d_{6}$-DMSO) of $\left[\mathrm{C}_{4} \mathrm{mim}\left[\mathrm{PF}_{6}\right]\right.$ after run 13 in biphasic system (RTIL/ $\left.\mathrm{H}_{2} \mathrm{O}\right)$, using $\mathrm{K}_{3} \mathrm{Fe}(\mathrm{CN})_{6}$ as co-oxidant

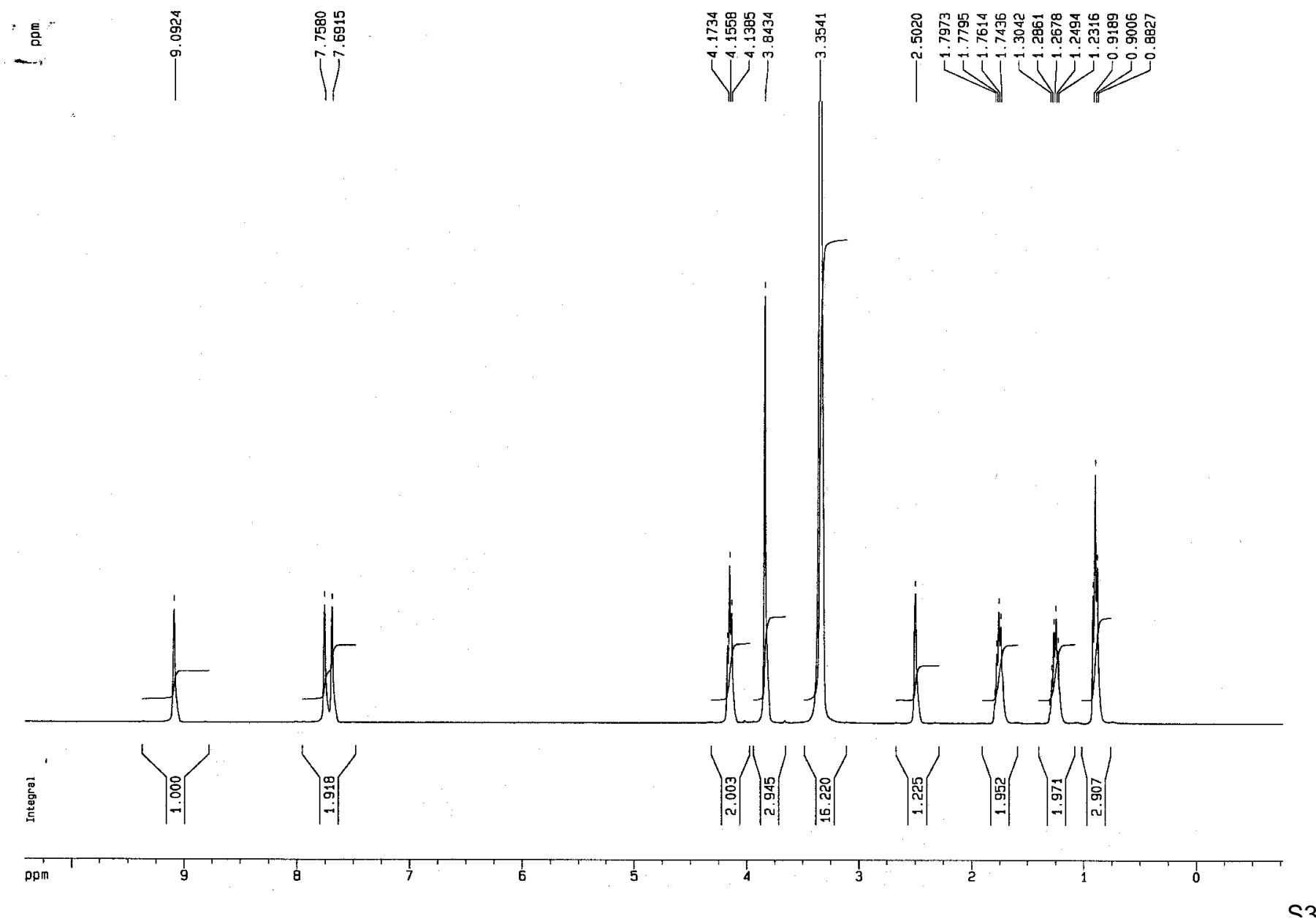


Figure 4. ${ }^{13} \mathrm{C}$ NMR spectra (in $d_{6}$ DMSO) of $\left[\mathrm{C}_{4} \mathrm{mim}\right]\left[\mathrm{PF}_{6}\right]$ after run 13 in biphasic system (RTIL/ $\mathrm{H}_{2} \mathrm{O}$ ), using $\mathrm{K}_{3} \mathrm{Fe}(\mathrm{CN})_{6}$ as co-oxidant

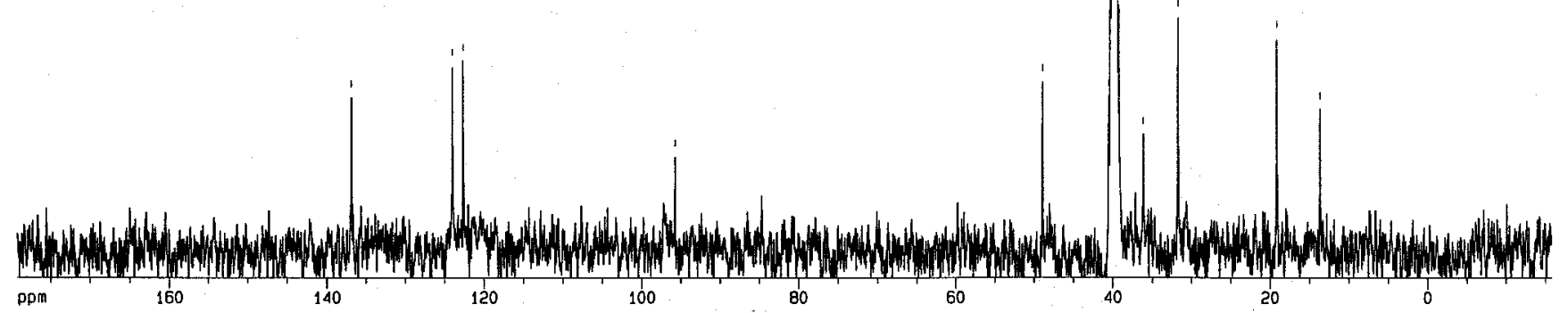

Figure 5. ${ }^{1} \mathrm{H}$ NMR spectra (in $d_{6}$-DMSO) of $\left[\mathrm{C}_{4} \mathrm{mim}\right]\left[\mathrm{PF}_{6}\right]$ after run 13 in monophasic system (RTIL/ $\left.\mathrm{H}_{2} \mathrm{O} / \mathrm{t}-\mathrm{BuOH}\right)$, using $\mathrm{K}_{3} \mathrm{Fe}(\mathrm{CN})_{6}$ as co-oxidant

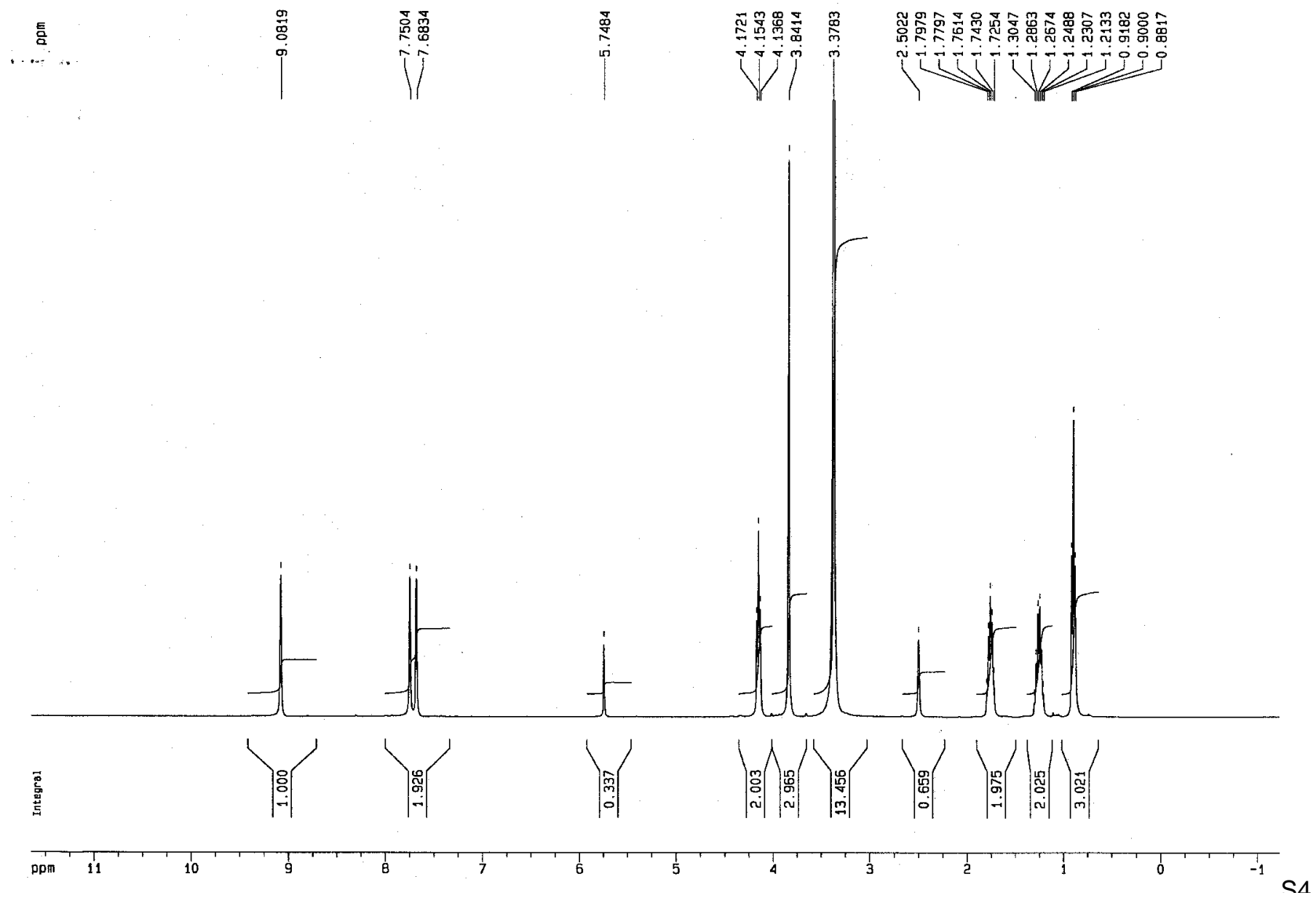


Figure 6. ${ }^{13} \mathrm{C}$ NMR spectra (in $d_{6}$-DMSO) of $\left[\mathrm{C}_{4} \mathrm{mim}\right]\left[\mathrm{PF}_{6}\right]$ after run 13 in monophasic system (RTIL/ $\left.\mathrm{H}_{2} \mathrm{O} / \mathrm{t}-\mathrm{BuOH}\right)$, using $\mathrm{K}_{3} \mathrm{Fe}(\mathrm{CN})_{6}$ as co-oxidant

言

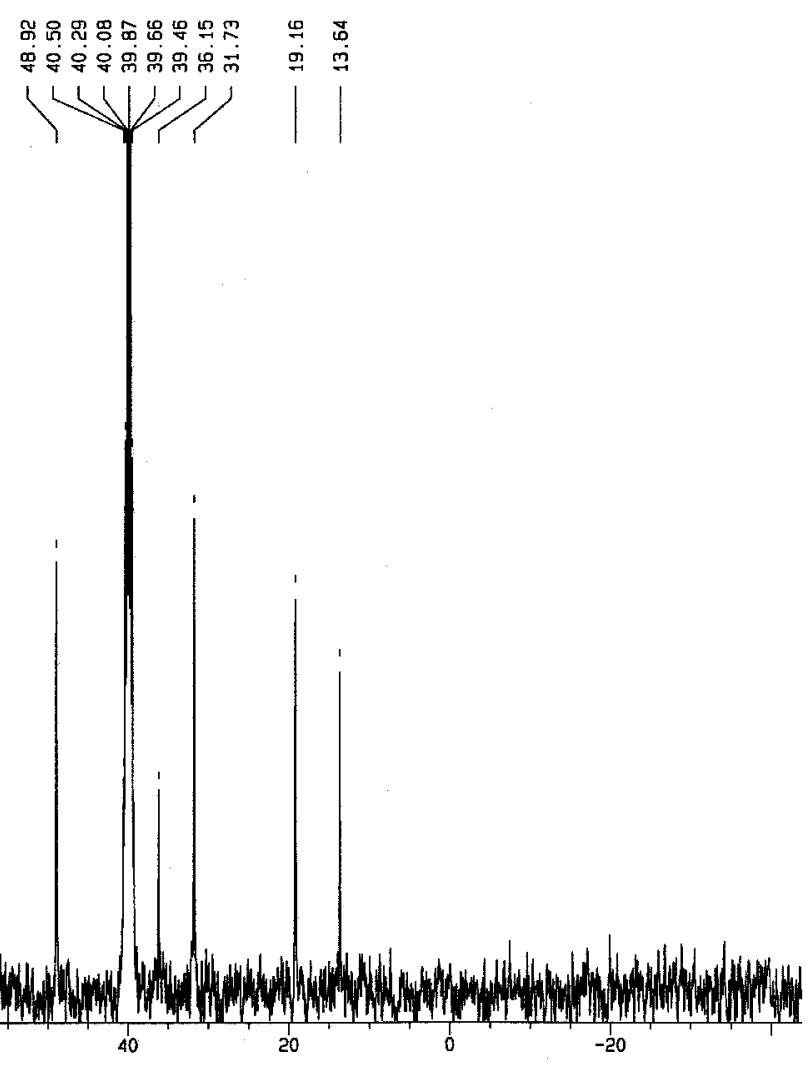

Figure 7. ${ }^{1} \mathrm{H}$ NMR spectra (in $d_{6}$-DMSO) of initial $\left[\mathrm{C}_{8} \mathrm{mim}\left[\mathrm{PF}_{6}\right]\right.$
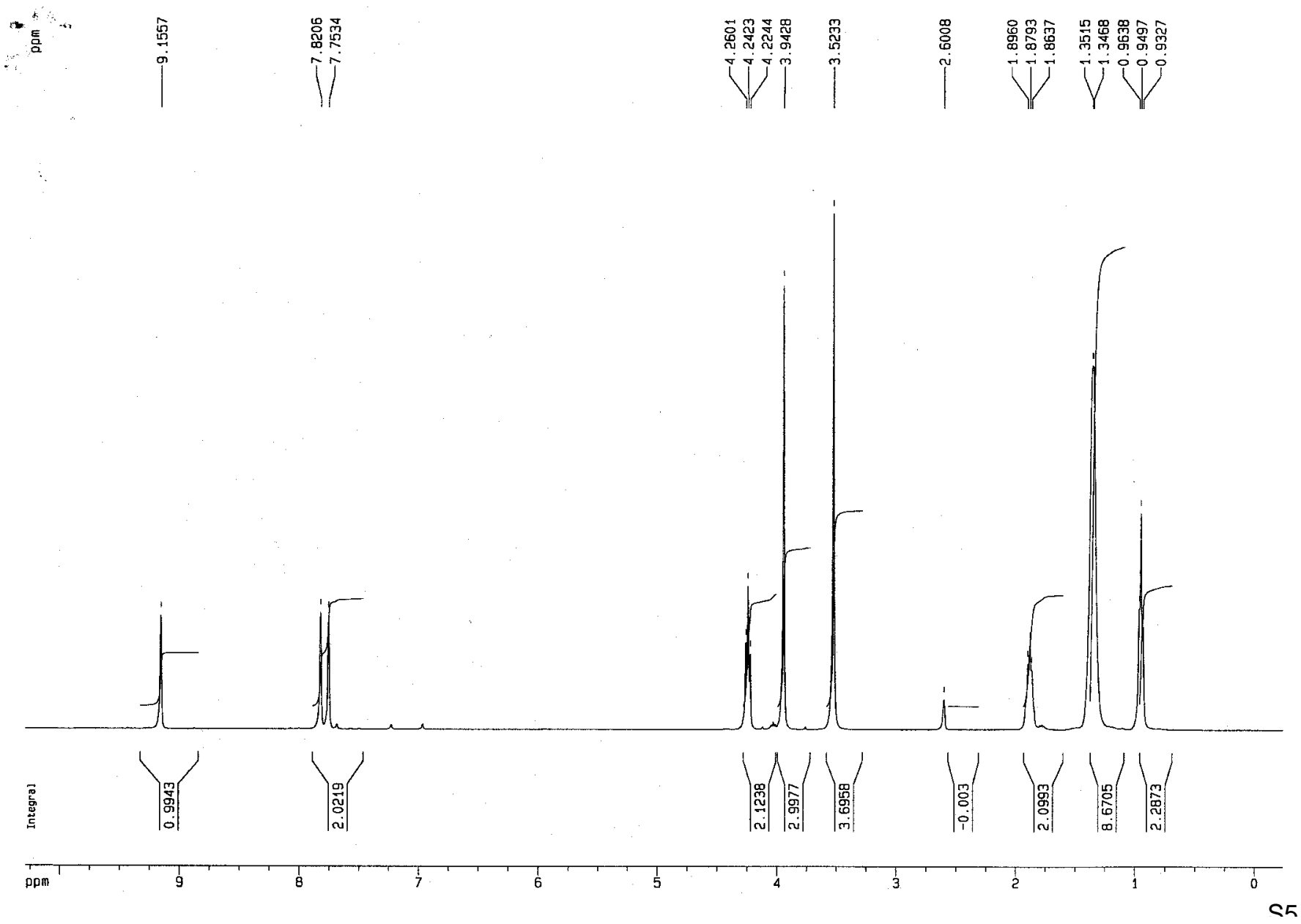
Figure 8. ${ }^{13} \mathrm{C}$ NMR spectra (in $d_{6}$-DMSO) of initial $\left[\mathrm{C}_{8}\right.$ mim] $\left[\mathrm{PF}_{6}\right]$

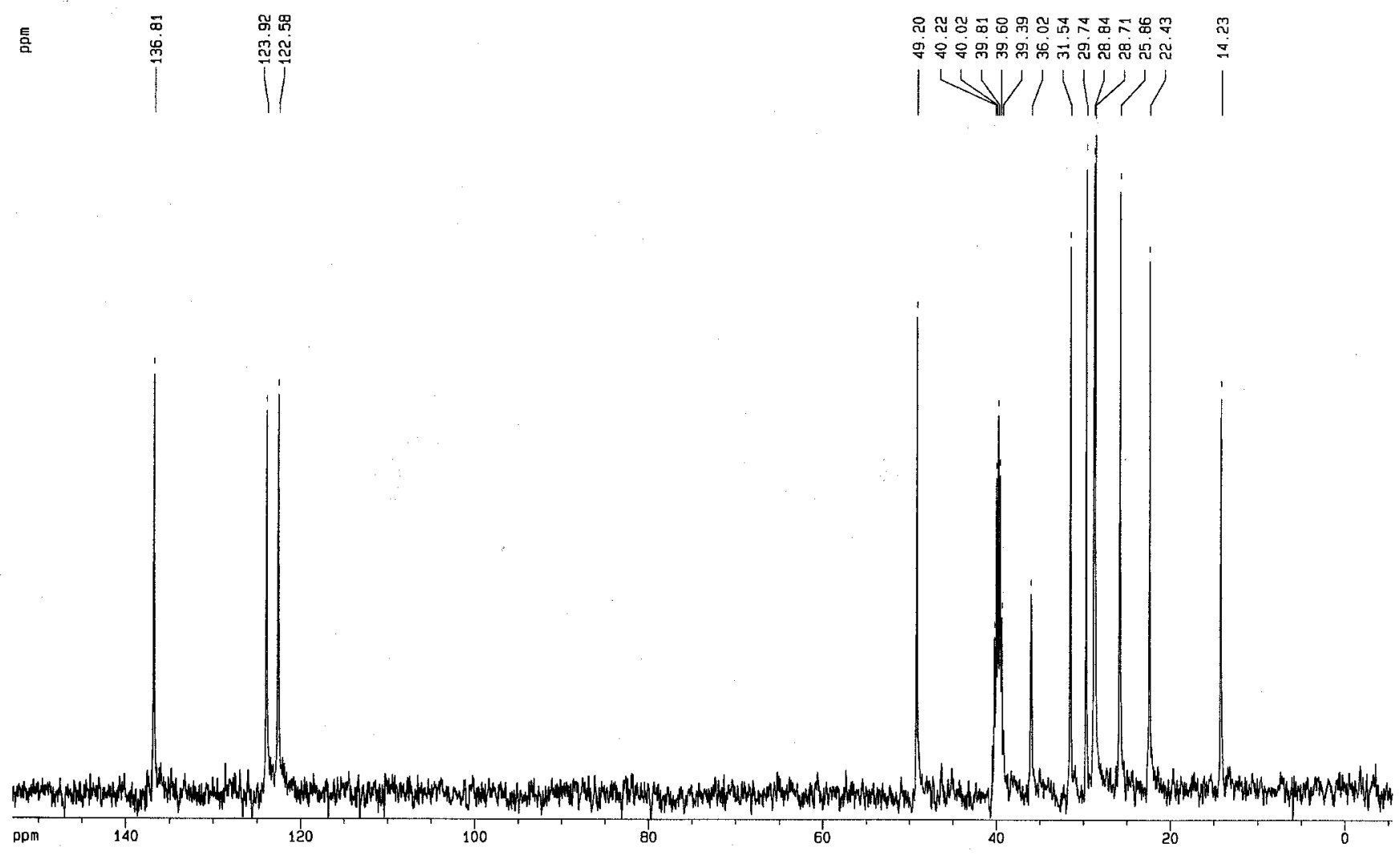

Figure 9. ${ }^{1} \mathrm{H}$ NMR spectra (in $d_{6}$-DMSO) of $\left[\mathrm{C}_{8} \mathrm{mim}\right]\left[\mathrm{PF}_{6}\right]$ after run 13 in biphasic system (RTIL/ $\left.\mathrm{H}_{2} \mathrm{O}\right)$, using $\mathrm{K}_{3} \mathrm{Fe}(\mathrm{CN})_{6}$ as co-oxidant

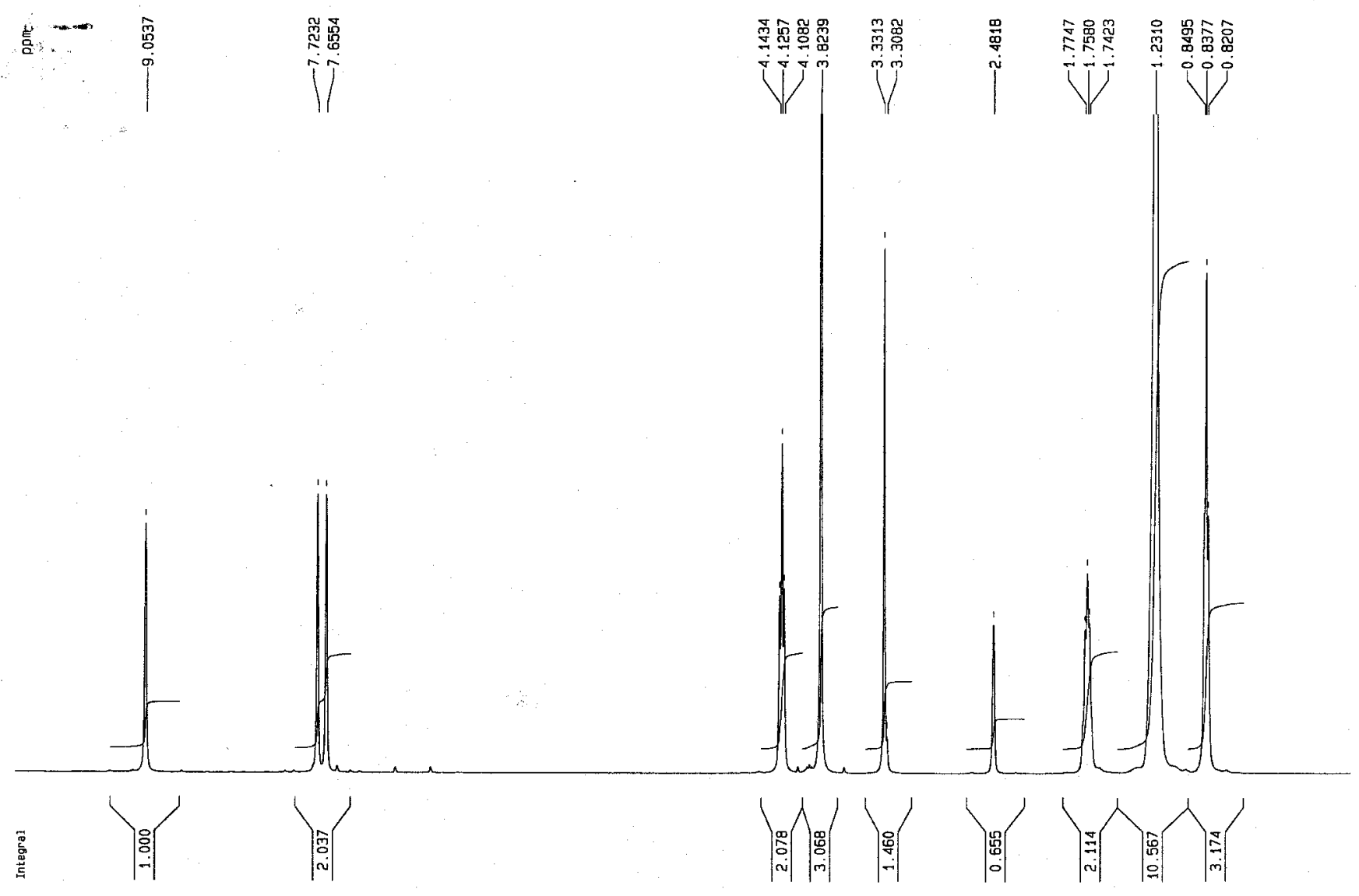


Figure 10. ${ }^{13} \mathrm{C}$ NMR spectra (in $d_{6}$ DMSO) of [ $\mathrm{C}_{8} \mathrm{mim}^{\mathrm{DM}}\left[\mathrm{PF}_{6}\right]$ after run 13 in biphasic system (RTIL/ $\mathrm{H}_{2} \mathrm{O}$ ), using $\mathrm{K}_{3} \mathrm{Fe}(\mathrm{CN})_{6}$ as co-oxidant

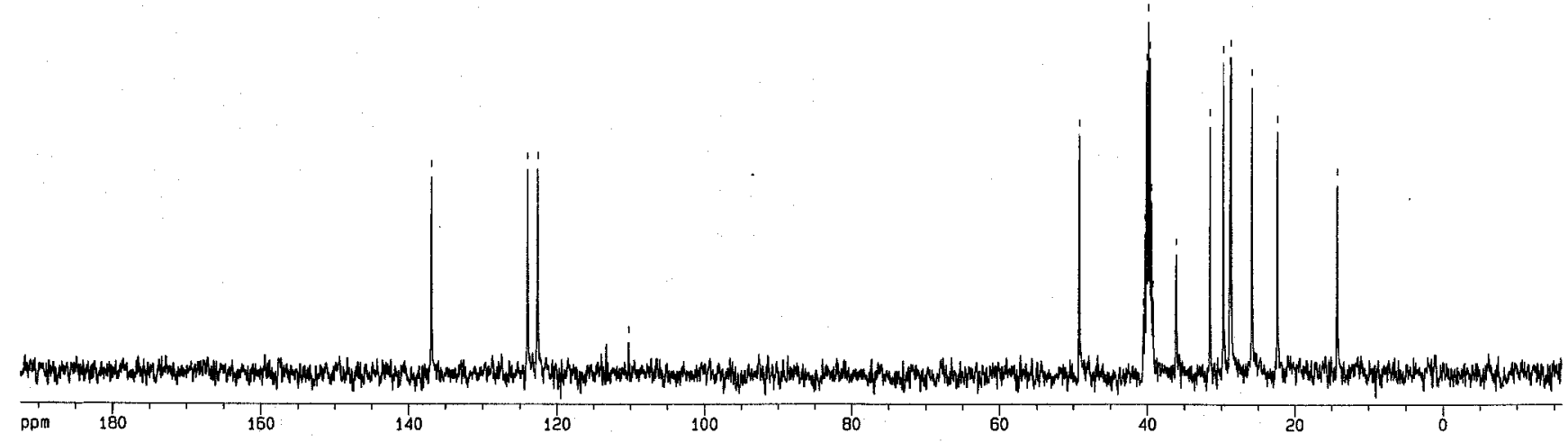

Figure 11. ${ }^{1} \mathrm{H}$ NMR spectra (in $d_{6} \mathrm{DMSO}$ ) of $\left[\mathrm{C}_{8} \mathrm{mim}\right]\left[\mathrm{PF}_{6}\right]$ after run 13 in monophasic system (RTIL/ $\left.\mathrm{H}_{2} \mathrm{O} / \mathrm{t}-\mathrm{BuOH}\right)$, using $\mathrm{K}_{3} \mathrm{Fe}(\mathrm{CN})_{6}$ as co-oxidant

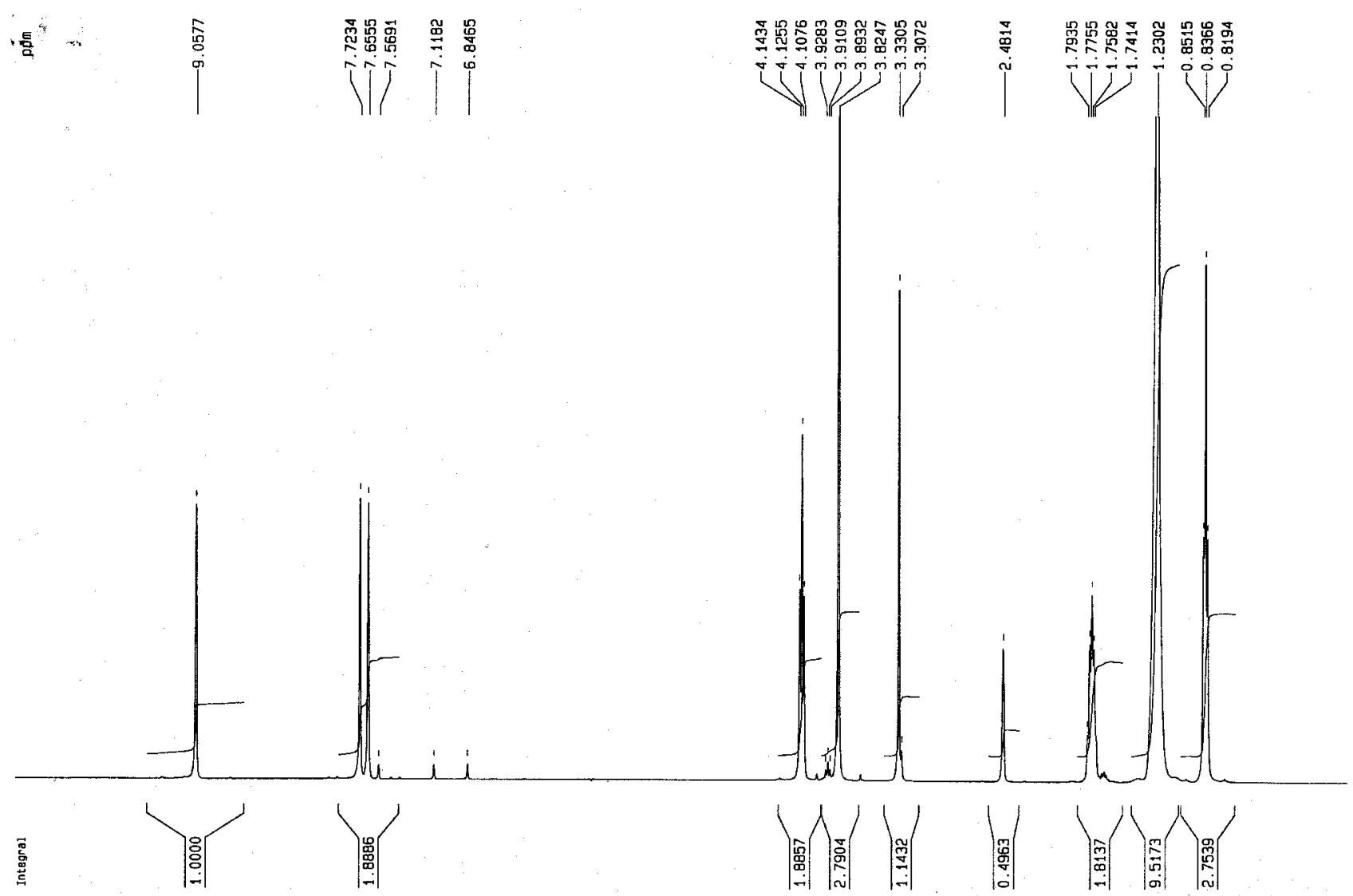


Figure 12. ${ }^{13} \mathrm{C}$ NMR spectra (in $d_{6}$-DMSO) of [ $\left.\mathrm{C}_{8} \mathrm{mim}\right]\left[\mathrm{PF}_{6}\right]$ after run 13 in monophasic system (RTIL/ $\left.\mathrm{H}_{2} \mathrm{O} / \mathrm{t}-\mathrm{BuOH}\right)$, using $\mathrm{K}_{3} \mathrm{Fe}(\mathrm{CN})_{6}$ as co-oxidant

言

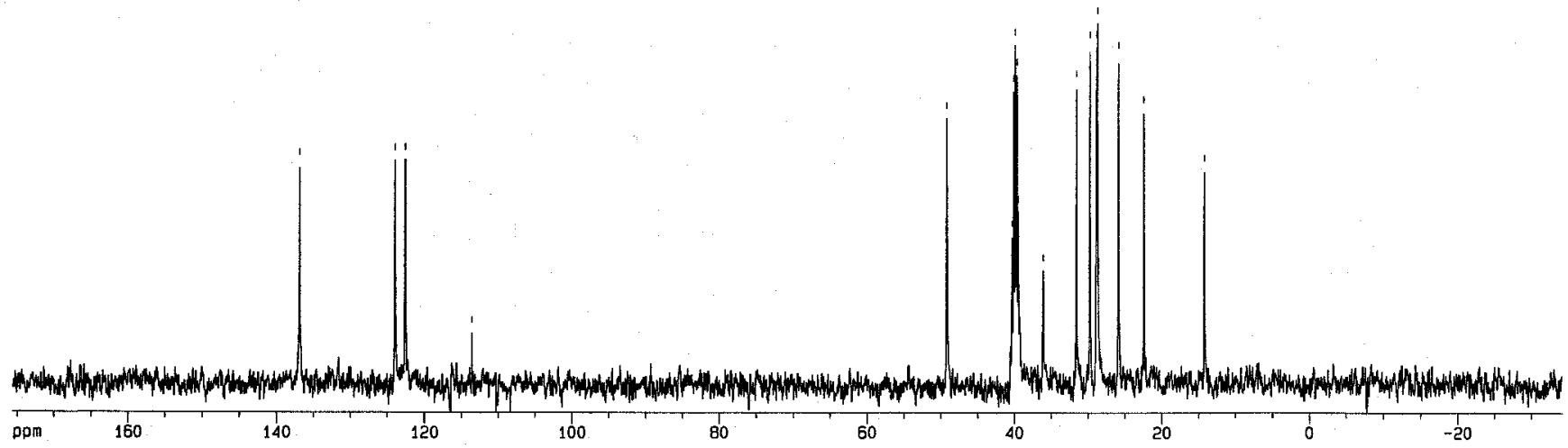

Figure 13. ${ }^{1} \mathrm{H}$ NMR spectra (in $d_{6}$-DMSO) of $\left[\mathrm{C}_{4}\right.$ mim] $\left[\mathrm{PF} \mathrm{F}_{6}\right]$ after run 13 in biphasic system (RTIL/ $\mathrm{H}_{2} \mathrm{O}$ ), using $\mathrm{NMO}$ as co-oxidant 
Figure 14. ${ }^{13} \mathrm{C}$ NMR spectra (in $d_{6}$ DMSO) of $\left[\mathrm{C}_{4} \mathrm{mim} \mathrm{DMF} \mathrm{PF}_{6}\right]$ after run 13 in biphasic system (RTIL/ $\mathrm{H}_{2} \mathrm{O}$ ), $\mathrm{NMO}$ as co-oxidant

\section{言}

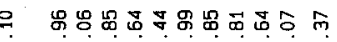

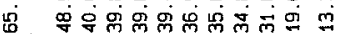

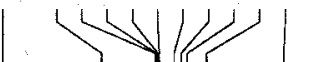

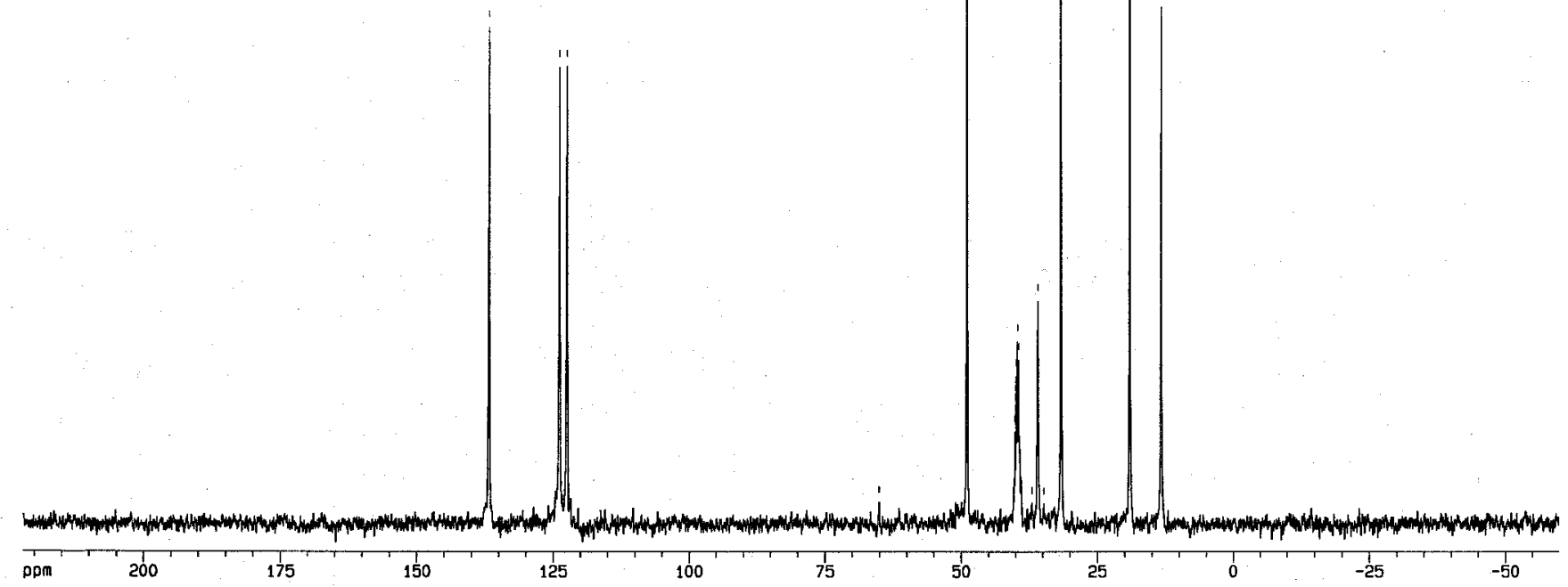

Figure 15. ${ }^{1} \mathrm{H}$ NMR spectra (in $d_{6}$-DMSO) of $\left[\mathrm{C}_{4} \mathrm{mim}\right]\left[\mathrm{PF}_{6}\right]$ after run 13 in monophasic system $\left(\mathrm{RTIL} / \mathrm{H}_{2} \mathrm{O} / \mathrm{t}-\mathrm{BuOH}\right)$, using $\mathrm{NMO}$ as co-oxidant
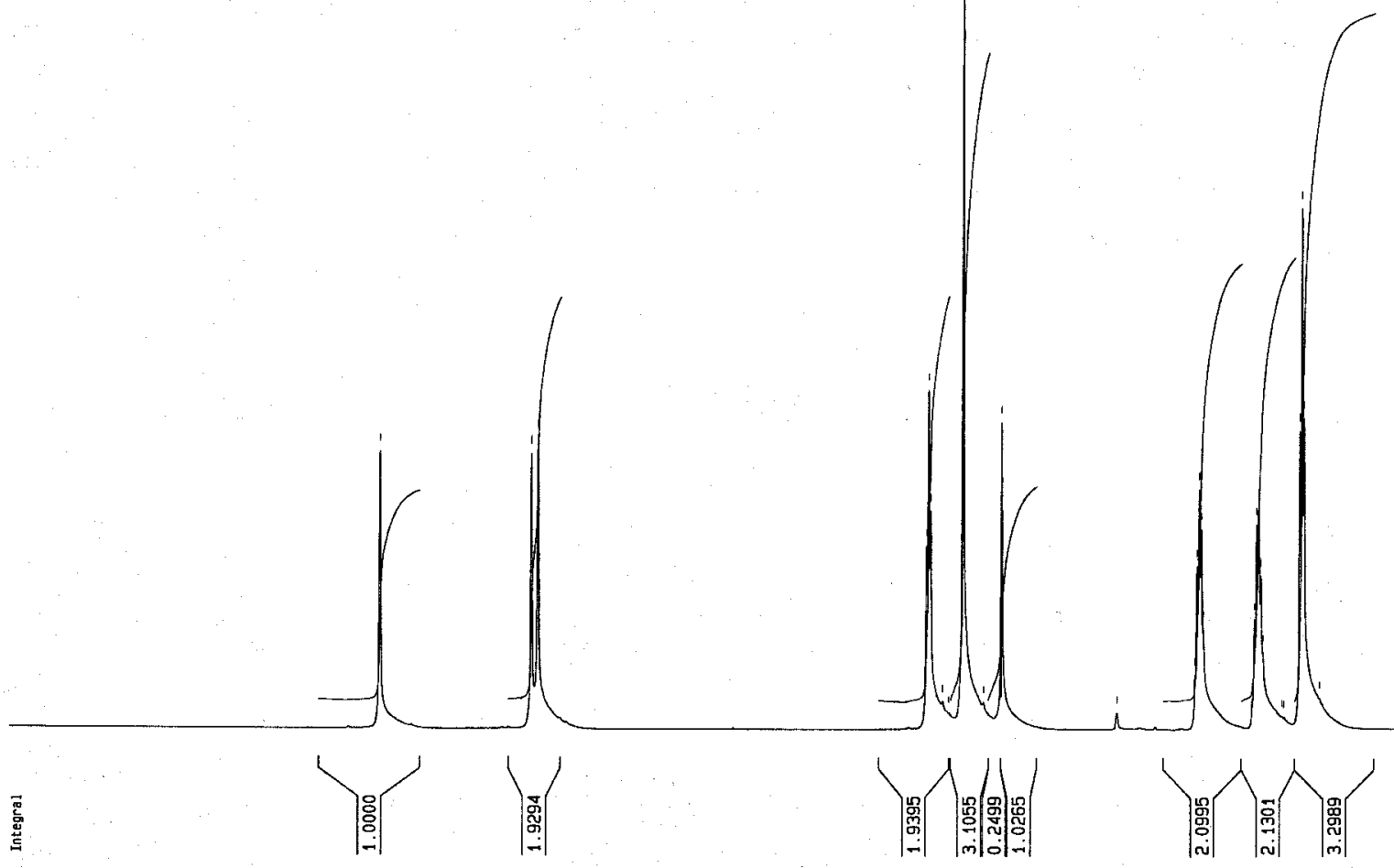
Figure 16. ${ }^{13} \mathrm{C}$ NMR spectra (in $d_{6}$-DMSO) of $\left[\mathrm{C}_{4} \mathrm{mim}\right]\left[\mathrm{PF}_{6}\right]$ after run 13 in monophasic system $\left(\mathrm{RTIL} / \mathrm{H}_{2} \mathrm{O} / \mathrm{t}-\mathrm{BuOH}\right)$, using $\mathrm{NMO}$ as co-oxidant

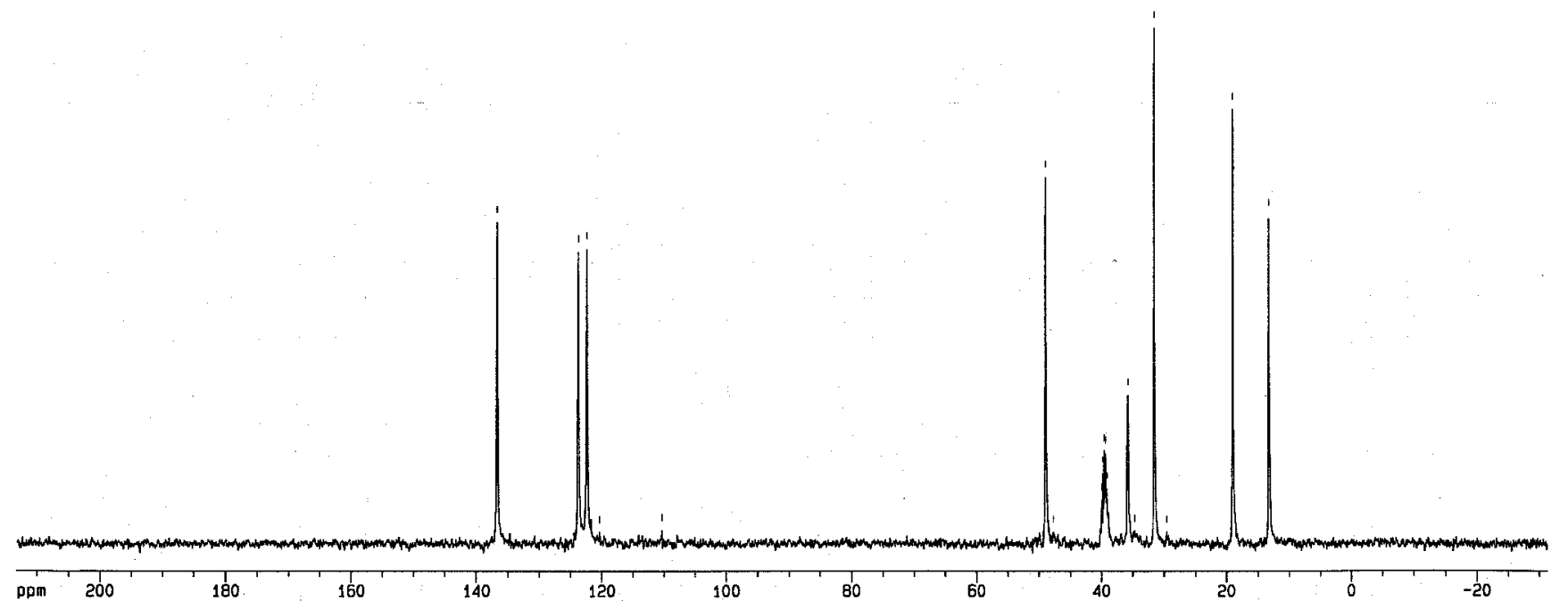

